\title{
Stable limits for associated regularly varying sequences
}

\author{
Adam Jakubowski* \\ Nicolaus Copernicus University, Poland \\ Dedicated to Professor Vygantas Paulauskas
}

\begin{abstract}
For a stationary sequence that is regularly varying and associated we give conditions which guarantee that partial sums of this sequence, under normalization related to the exponent of regular variation, converge in distribution to a stable, non-Gaussian limit. The obtained limit theorem admits a natural extension to the functional convergence in Skorokhod's $M_{1}$ topology.
\end{abstract}

Keywords: stable laws, association, regular variation, limit theorems, Skorokhod's $M_{1}$ topology.

MSClassification 2010: 60F05, 60F17, 60E07, 60E15, 60G10.

\section{Introduction}

In the pioneering work [17] Paulauskas considered some covariance-like quantities, which were defined for jointly stable random variables. He returned to this topic in recent papers [7] and [8] with a variety of examples based on linear processes with heavy tailed innovations (see also [18] and [19]). It is clear by now that these various quantities are very useful in limit theorems.

Perhaps most elegant example of limit theorems operating with covariance-like quantities was given in [6] for associated and jointly stable stationary sequences (see also [8] for generalization of these results to stationary random fields).

Recall (see e.g. [23]) that random variables $X_{1}, X_{2}, \ldots$ are jointly $\alpha$-stable, $0<\alpha<2$, if for each $n \in \mathbb{N}$ there exists a finite Borel measure $\Gamma_{n}$ on the unit sphere in $\mathbb{R}^{n}$,

$$
\mathcal{S}^{n-1}=\left\{\mathbf{s}=\left(s_{1}, \ldots s_{n}\right) \in \mathbb{R}^{n}: \sum_{i=1}^{n} s_{i}^{2}=1\right\}
$$

and a vector $\mathbf{b}_{n} \in \mathbb{R}^{n}$ such that the characteristic function of $\mathbf{X}_{n}=\left(X_{1}, X_{2}, \ldots, X_{n}\right)$ is of the form

$$
E \exp i\left(\mathbf{t}, \mathbf{X}_{n}\right)=\exp \left(i\left(\mathbf{b}_{n}, \mathbf{t}\right)+\int_{\mathcal{S}^{n-1}} \int_{0}^{\infty} g(\mathbf{t}, \mathbf{s}, r) \frac{d r}{r^{\alpha+1}} \Gamma_{n}(d \mathbf{s})\right) .
$$

*E-mail: adjakubo@mat.umk.pl 
Here

$$
g(\mathbf{t}, \mathbf{s}, r)= \begin{cases}e^{i(\mathbf{t}, \mathbf{s}) r}-1, & \text { if } 0<\alpha<1, \\ e^{i(\mathbf{t}, \mathbf{s}) r}-1-i(\mathbf{t}, \mathbf{s}) r I(r \leq 1), & \text { if } \alpha=1, \\ e^{i(\mathbf{t}, \mathbf{s}) r}-1-i(\mathbf{t}, \mathbf{s}) r, & \text { if } 1<\alpha<2 .\end{cases}
$$

We will write $\mathcal{L}\left(\mathbf{X}_{n}\right)=\gamma_{\alpha}\left(\mathbf{b}_{n}, \Gamma_{n}\right)$. Clearly, if $X_{1}, X_{2}, \ldots$ are also strictly stationary, then for some $b \in \mathbb{R}^{1}$

$$
\mathbf{b}_{n}=(\underbrace{b, \ldots, b}_{n \text { times }}), \quad n \in \mathbb{N} .
$$

For one-dimensional stable distributions we have $\mathcal{S}^{0}=\{-1,1\}$, and we shall use the notation

$$
\gamma_{\alpha}(b, \Gamma(\{1\}), \Gamma(\{-1\})) \equiv \gamma_{\alpha}(b, \Gamma) .
$$

Jointly stable random variables $X_{1}, X_{2}, \ldots$ are strictly $\alpha$-stable, if either

(a) $\mathbf{b}_{n}=0, n \in \mathbb{N}$, when $\alpha \neq 1$, or

(b) $\int_{\mathcal{S}^{n-1}} \mathbf{s} \Gamma_{n}(d \mathbf{s})=0, n \in \mathbb{N}$, when $\alpha=1$. This holds if, for instance, $\Gamma_{n}$ is a symmetric measure on $\mathcal{S}^{n-1}$.

The other key assumption in this paper is association. Following [9] we call random variables $X_{1}, X_{2}, \ldots, X_{n}$ associated if

$$
\operatorname{Cov}\left(f\left(X_{1}, X_{2}, \ldots, X_{n}\right), g\left(X_{1}, X_{2}, \ldots, X_{n}\right)\right) \geq 0,
$$

for each pair of functions $f, g: \mathbb{R}^{n} \rightarrow \mathbb{R}^{1}$, which are non-decreasing in each coordinate and for which the above covariance exists. An infinite collection of random variables is associated, if its every finite subset consists of associated random variables. For basic properties of associated random variables we refer to the original article [9] and also to the more recent source [4].

For jointly $\alpha$-stable random variables there exists an astonishingly simple description of association, due to Lee, Rachev and Samorodnitsky [13] (see also [21], 22] and [1] for related results). The measure $\Gamma_{n}$ has to be concentrated on "positive" and "negative" parts of $\mathcal{S}^{n-1}$, i.e.

$$
\Gamma_{n}\left(\mathcal{S}^{n-1} \cap\left\{[0,+\infty)^{n} \cup(-\infty, 0]^{n}\right\}^{c}\right)=0 .
$$

This property allows proving very nice limit theorems, which we restate here from [6] as Theorems 1-3, for they will be used in the course of the proofs of our new, more general result.

In what follows $S_{n}$ will always stand for $X_{1}+X_{2}+\ldots+X_{n}$.

As in the case of independent summands, we have separate results for the three cases where $0<\alpha<1, \alpha=1$, and $1<\alpha<2$.

Theorem 1. Let $X_{1}, X_{2}, \ldots$ be stationary, associated and jointly $\alpha$-stable, $0<\alpha<1$. Then

$$
\frac{S_{n}}{n^{1 / \alpha}} \underset{\mathcal{D}}{\longrightarrow} \mu_{\infty}
$$

where $\mu_{\infty}$ is a strictly $\alpha$-stable distribution.

Theorem 2. Let $X_{1}, X_{2}, \ldots$ be stationary, associated and jointly 1-stable. Then there exist constants $A_{n}$ such that

$$
\frac{S_{n}}{n}-A_{n} \sim X_{1} .
$$


In particular, if $\Gamma_{n}$ is symmetric for each $n \in \mathbb{N}$, then

$$
\frac{S_{n}}{n} \sim X_{1}, \quad n \in \mathbb{N} .
$$

Theorem 3. Let $X_{1}, X_{2}, \ldots$ be stationary, associated and jointly $\alpha$-stable, $1<\alpha<2$, with two-dimensional distributions $\mathcal{L}\left(\left(X_{1}, X_{k}\right)\right)=\gamma_{\alpha}\left((b, b), \Gamma_{\{1, k\}}\right)$.

If

$$
\sum_{k=2}^{\infty} \int_{\mathcal{S}^{1}} s_{1} s_{2} \Gamma_{\{1, k\}}(d \mathbf{s})<+\infty,
$$

then

$$
\frac{S_{n}-E S_{n}}{n^{1 / \alpha}}=\frac{S_{n}-n b}{n^{1 / \alpha}} \underset{\mathcal{D}}{\longrightarrow} \mu_{\infty}
$$

where $\mu_{\infty}$ is a non-degenerate strictly $\alpha$-stable distribution.

Notice that Theorem 3 convincingly supports the point of view of [17] that the spectral covariance

$$
\int_{\mathcal{S}^{1}} s_{1} s_{2} \Gamma_{\{1, k\}}(d \mathbf{s})
$$

is a covariance-like quantity. To see this let us compare the shape of Theorem 3 with the classic central limit theorem for associated stationary sequences due to Newman [15] (see also [16] for functional convergence and [5] for a more general result): suppose that $\left\{X_{j}\right\}$ is stationary and associated and that $E X_{1}=0, E X_{1}^{2}<+\infty$. Then condition

$$
\sigma^{2}=E X_{1}^{2}+2 \sum_{j=2}^{\infty} E X_{1} X_{j}<+\infty
$$

implies $S_{n} / \sqrt{n} \longrightarrow_{\mathcal{D}} \mathcal{N}\left(0, \sigma^{2}\right)$, as $n \rightarrow \infty$.

Of course the assumption on joint $\alpha$-stability appearing in Theorems 1, 2 and 3 is very restrictive. Theorem 2.8 in [6] gets rid of this limitation and introduces another covariance-like quantity.

For a strictly stationary sequence $\left\{X_{j}\right\}_{j \in \mathbb{N}}$ define

$$
H_{\left(X_{i}, X_{j}\right)}\left(x_{i}, x_{j}\right)=P\left(X_{i} \leq x_{i}, X_{j} \leq x_{j}\right)-P\left(X_{i} \leq x_{i}\right) P\left(X_{j} \leq x_{j}\right) .
$$

Then fix $A>0$ and $\alpha \in(0,2)$ and define

$$
I_{\alpha}^{A}\left(X_{i}, X_{j}\right)=\sup _{a \geq A} a^{p-2} \int_{-a}^{a} \int_{-a}^{a} H_{\left(X_{i}, X_{j}\right)}(x, y) d x d y .
$$

It is immediate that if $\left\{X_{j}\right\}$ is associated, then both $H_{\left(X_{i}, X_{j}\right)}\left(x_{i}, x_{j}\right) \geq 0$ and $I_{\alpha}^{A}\left(X_{i}, X_{j}\right) \geq 0$, with the latter taking possibly the value $+\infty$. The quantity $I_{\alpha}^{A}\left(X_{i}, X_{j}\right)$ satisfies the CauchySchwarz inequality:

$$
I_{\alpha}^{A}\left(X_{i}, X_{j}\right) \leq \sqrt{I_{\alpha}^{A}\left(X_{i}, X_{i}\right)} \sqrt{I_{\alpha}^{A}\left(X_{j}, X_{j}\right)} .
$$

Moreover, as [6, Theorem 2.8] states, condition

$$
\sum_{k=2}^{\infty} I_{\alpha}^{A}\left(X_{1}, X_{k}\right)<+\infty
$$


plus some natural distributional conditions imply convergence of partial sums to stable laws.

The serious drawback of coefficient $I_{\alpha}^{A}\left(X_{i}, X_{j}\right)$ is that it is infinite for some marginal distributions $\mathcal{L}\left(X_{i}\right)$ belonging to the domain of attraction of a stable law.

In the present paper we give assumptions which are the most general when considering the framework based on domains of attraction and Newman's inequality.

\section{Statement of results}

Let $\left\{X_{j}\right\}$ be a stationary sequence. We will say that it is regularly varying, if it is jointly regularly varying with some index $\alpha$, i.e. for each $i \leq j$ the joint distribution of $\left(X_{i}, X_{i+1}, \ldots, X_{j}\right)$ is regularly varying with necessarily the same index $\alpha$. We will use a reformulation of regular variation, which is close in spirit to [3, Theorem 2.1] and can be proved in a similar way (see also [20]).

In what follows we shall assume that

$$
\mathbb{P}\left(\left|X_{1}\right|>x\right)=x^{-\alpha} \ell(x),
$$

where $\alpha \in(0,2)$ and $\ell(x)$ is a slowly varying function. Given (15) we define the normalizing constants $B_{n}$ by the relation

$$
n \mathbb{P}\left(\left|X_{1}\right|>B_{n}\right) \rightarrow 1 .
$$

It is well-known that $\left\{B_{n}\right\}$ is $1 / \alpha$-regularly varying.

For multidimensional distributions we assume that for each $N \in \mathbb{N}$

$$
n \mathbb{P}\left(\left(\frac{X_{1}}{B_{n}}, \frac{X_{2}}{B_{n}}, \ldots, \frac{X_{N}}{B_{n}}\right) \in(\cdot)\right) \longrightarrow \nu_{N}(\cdot), \text { vaguely on } \overline{\mathbb{R}}^{m} \backslash\{\mathbf{0}\},
$$

where $\nu_{N}$ is (necessarily) a Lévy measure on $\mathbb{R}^{N}$.

In addition we always assume that

if $\alpha=1$, then vectors $\left(X_{1}, X_{2}, \ldots, X_{N}\right)$ have symmetric distributions, $N \in \mathbb{N}$,

if $\alpha \in(1,2)$, then $\mathbb{E} X_{1}=0$.

Let us observe that our assumptions (15) - (19) imply that for each $N \in \mathbb{N}$

$$
\frac{\mathbf{Z}_{N, 1}+\mathbf{Z}_{N, 2}+\ldots+\mathbf{Z}_{N, n}}{B_{n}} \underset{\mathcal{D}}{\longrightarrow}\left(Y_{1}^{N}, Y_{2}^{N}, \ldots, Y_{N}^{N}\right)
$$

where $\mathbf{Z}_{N, 1}, \mathbf{Z}_{N, 2}, \ldots, \mathbf{Z}_{N, n}, \ldots$ are independent copies of $\left(X_{1}, X_{2}, \ldots, X_{N}\right)$ and $\left(Y_{1}^{N}, \ldots, Y_{N}^{N}\right)$ is a strictly $\alpha$-stable random vector with the distribution determined by $\nu_{N}$.

For fixed $a>0$ we define a function $f_{a}: \mathbb{R}^{1} \rightarrow \mathbb{R}^{1}$ by

$$
f_{a}(x)=\left\{\begin{aligned}
a & \text { if } x>a \\
x & \text { if }|x| \leq a \\
-a & \text { if } x<-a
\end{aligned}\right.
$$

Note that $f_{a}(x / b)=b^{-1} f_{a b}(x)$, that $f_{a}(x)$ is a non-decreasing function in $x$, and that $\left\{f_{a}\left(X_{j}\right): j \geq 1\right\}$ is again an associated sequence of random variables. Moreover, $f_{a}(x)$ is absolutely continuous with $f_{a}^{\prime}(x)=I_{(-a, a)}(x)$ a.e. and so, by [26, Lemma 3.1]

$$
\operatorname{Cov}\left(f_{a}\left(X_{i}\right), f_{a}\left(X_{j}\right)\right)=\int_{-a}^{a} \int_{-a}^{a} H_{\left(X_{i}, X_{j}\right)}\left(x_{i}, x_{j}\right) d x_{i} d x_{j}
$$


It follows from (20) that for each $N \geq 2$ and every $a>0$

$$
\begin{aligned}
n \cdot B_{n}^{-2} \operatorname{Cov}\left(f_{a \cdot B_{n}}\left(X_{1}\right), f_{a \cdot B_{n}}\left(X_{N}\right)\right)=n \cdot \operatorname{Cov}\left(f_{a}\left(\frac{X_{1}}{B_{n}}\right), f_{a}\left(\frac{X_{N}}{B_{n}}\right)\right. & \\
& \underset{n \rightarrow \infty}{\longrightarrow} \int_{\mathbb{R}^{2}} f_{a}\left(x_{1}\right) f_{a}\left(x_{2}\right) \nu_{\{1, N\}}\left(d x_{1}, d x_{2}\right),
\end{aligned}
$$

where $\nu_{\{1, N\}}\left(d x_{1}, d x_{2}\right)$ is the Lévy measure of $\mathcal{L}\left(Y_{1}^{N}, Y_{N}^{N}\right)$ (see e.g. [12, Theorem 2.35, p.362]). This implies that for each $N \geq 2$ the non-decreasing function

$$
a \mapsto g_{N}(a)=\operatorname{Cov}\left(f_{a}\left(X_{1}\right), f_{a}\left(X_{N}\right)\right)
$$

is regularly varying and the exponent of regular variation is $2-\alpha$ (see e.g. [10, Lemma 3 , p. $277]$ ).

In fact our main assumption requires substantially more:

$$
a \mapsto \sum_{j=2}^{\infty} g_{j}(a) \text { is a regularly varying function. }
$$

We relate this abstract property with (23) by assuming

$$
n \sum_{j=2}^{\infty} \operatorname{Cov}\left(f_{1}\left(\frac{X_{1}}{B_{n}}\right), f_{1}\left(\frac{X_{j}}{B_{n}}\right)\right) \underset{n \rightarrow \infty}{\longrightarrow} \sum_{j=2}^{\infty} \int_{\mathbb{R}^{2}} f_{1}\left(x_{1}\right) f_{1}\left(x_{2}\right) \nu_{\{1, j\}}\left(d x_{1}, d x_{2}\right)<+\infty .
$$

Both (24) and (25) imply that $\sum_{j=2}^{\infty} g_{j}(a)$ is $(2-\alpha)$-regularly varying.

Remark 1. Assumptions (24) and (25) taken together are equivalent to

$$
n \sum_{j=2}^{\infty} \operatorname{Cov}\left(f_{a}\left(\frac{X_{1}}{B_{n}}\right), f_{a}\left(\frac{X_{j}}{B_{n}}\right)\right) \underset{n \rightarrow \infty}{\longrightarrow} \sum_{j=2}^{\infty} \int_{\mathbb{R}^{2}} f_{a}\left(x_{1}\right) f_{a}\left(x_{2}\right) \nu_{\{1, j\}}\left(d x_{1}, d x_{2}\right)<+\infty, \quad a>0 .
$$

As the limit is continuous and monotone in $a$, the above pointwise convergence is, in fact, uniform on bounded intervals.

Theorem 4. Let $\left\{X_{j}\right\}$ be a stationary sequence that is associated and satisfies conditions (15) - (19).

Suppose that (24) and (25) hold with $B_{n}$ defined by (16).

Then there exists a strictly $\alpha$-stable distribution $\mu_{\infty}$ such that

$$
\frac{X_{1}+X_{2}+\ldots+X_{n}}{B_{n}} \underset{\mathcal{D}}{\longrightarrow} \mu_{\infty}
$$

Remark 2. Theorem 2.13 in [6] operates with apparently weaker assumption on domain of attraction for sums of $S_{N}, N \in \mathbb{N}$, only. It is not clear whether assumptions of this type are weaker or equivalent to our conditions (15) - (19). See [2] for discussion of problems of similar flavor.

Remark 3. As Remark 2.4 in [6] shows, if $\alpha \in(0,1)$ then there are jointly stable associated sequences such that the limit is degenerate. We think that in the presence of the strong assumption of summability (26) it is possible to show the non-degeneracy of the limit. We are, however, not able to prove this statement. 
Theorem 4 admits a natural functional extension. Let us define a sequence of stochastic processes with trajectories in the Skorokhod space $\mathbb{D}\left([0,1]: \mathbb{R}^{1}\right)$.

$$
S_{n}(t)=\frac{S_{\lfloor n \cdot t\rfloor}}{B_{n}}, \quad t \in[0,1] .
$$

It is known ([1]) that in the general setting of associated sequences it is impossible to obtain the convergence of $\left\{S_{n}(t)\right\}$ in Skorokhod's $J_{1}$ topology. On the other hand, applying the powerful Theorem 1 of [14], we shall obtain the convergence in Skorokhod's $M_{1}$ topology. For the definitions and basic properties of Skorokhod's topologies we refer either to the seminal paper [24] or to the extensive source [25].

Theorem 5. In assumptions of Theorem 4, the sequence $\left\{S_{n}(t)\right\}$ converges in law on the Skorokhod space $\mathbb{D}\left([0,1]: \mathbb{R}^{1}\right)$ equipped with Skorokhod's $M_{1}$ topology. The limit is the stable Lévy process $\{Y(t)\}$ given by $Y(1) \sim \mu_{\infty}$.

\section{Proofs}

As noted in Introduction, we follow the line of the proof of Theorem 2.8 in $[6]$. But the details are different in many places, for our result is more general. Therefore we give here a complete proof.

\subsection{Proof of Theorem 4}

Let us recall (20), i.e. for each $N$

$$
\frac{\mathbf{Z}_{N, 1}+\mathbf{Z}_{N, 2}+\ldots+\mathbf{Z}_{N, n}}{B_{n}} \underset{\mathcal{D}}{\longrightarrow}\left(Y_{1}^{N}, Y_{2}^{N}, \ldots, Y_{N}^{N}\right)
$$

where $\mathbf{Z}_{N, 1}, \mathbf{Z}_{N, 2}, \ldots, \mathbf{Z}_{N, n}, \ldots$ are independent copies of $\left(X_{1}, X_{2}, \ldots, X_{N}\right)$. It follows that there exists a stationary process $\left\{Y_{j}\right\}$ such that

$$
\left(Y_{1}, Y_{2}, \ldots, Y_{N}\right) \sim\left(Y_{1}^{N}, Y_{2}^{N}, \ldots, Y_{N}^{N}\right), N \in \mathbb{N} .
$$

The process $\left\{Y_{j}\right\}$ is jointly $\alpha$-strictly stable and associated. We shall call $\left\{Y_{j}\right\}$ the stable tangent to $\left\{X_{j}\right\}$, for the asymptotic properties of partial sums of the original and the tangent processes are the same. Notice that for $\alpha \in[1,2)$ the existence of the tangent process requires more than just the regular variation of $\left\{X_{j}\right\}$, therefore we do not introduce here the tail process as defined in [3].

Let $\mu_{N}$ be the distribution of $Y_{1}+Y_{2}+\ldots+Y_{N}$. By the strict $\alpha$-stability

$$
\frac{Y_{1}+Y_{2}+\ldots+Y_{N}}{N^{1 / \alpha}} \sim \mu_{N}^{*(1 / N)}
$$

Here $\mu^{* \beta}$ is the convolution $\beta$-power of the infinitely divisible distribution $\mu$.

By the association and Theorems 1, 2 and 3 there exists a strictly $\alpha$-stable distribution $\mu_{\infty}$ such that as $N \rightarrow \infty$

$$
\mu_{N}^{*(1 / N)} \Longrightarrow \mu_{\infty}
$$

Notice that for $\alpha \in(1,2)$ relation (9) in Theorem [3] is satisfied by [6, Remark 2.6] and our assumption (25). 
It follows from (20) and (28) that for each $N \in \mathbb{N}$ and as $n \rightarrow \infty$

$$
\left(\mathbb{E} e^{i \lambda\left(X_{1}+X_{2}+\ldots+X_{N}\right) / N^{1 / \alpha} B_{n}}\right)^{n} \longrightarrow\left(\hat{\mu}_{N}(\lambda)\right)^{1 / N}, \lambda \in \mathbb{R}^{1} .
$$

By the regular variation of $\left\{B_{n}\right\}, N^{1 / \alpha} B_{n} \sim B_{N \cdot n}$, as $n \rightarrow \infty$, so the above relation can be rewritten as

$$
\left(\mathbb{E} e^{\left.i \lambda S_{N} / B_{n}\right)}\right)^{\lfloor n / N\rfloor} \longrightarrow\left(\hat{\mu}_{N}(\lambda)\right)^{1 / N}, N \in \mathbb{N}, \lambda \in \mathbb{R}^{1} .
$$

This and (29) imply $\lim _{N \rightarrow \infty} \lim _{n \rightarrow \infty}\left|\left(\mathbb{E} e^{\left.i \lambda S_{N} / B_{n}\right)}\right)^{\lfloor n / N\rfloor}-\hat{\mu}_{\infty}(\lambda)\right|=0, \lambda \in \mathbb{R}^{1}$. Therefore it is enough to prove that $\lim _{N \rightarrow \infty} \limsup _{n \rightarrow \infty}\left|\mathbb{E} e^{i \lambda\left(S_{n} / B_{n}\right)}-\left(E e^{i \lambda\left(S_{N} / B_{n}\right)}\right)^{\lfloor n / N\rfloor}\right|=0, \lambda \in \mathbb{R}^{1}$, or, after a simple modification,

$$
\lim _{N \rightarrow \infty} \limsup _{m \rightarrow \infty}\left|\mathbb{E} e^{i \lambda S_{m \cdot N} / B_{m \cdot N}}-\left(E e^{i \lambda S_{N} / B_{m \cdot N}}\right)^{m}\right|=0, \quad \lambda \in \mathbb{R}^{1} .
$$

Recall that function $f_{a}$ is defined by (21). Consider the following decomposition.

$$
B_{n}^{-1} \sum_{j=1}^{k} X_{j}=\sum_{j=1}^{k} f_{a}\left(B_{n}^{-1} X_{j}\right)+\sum_{j=1}^{k}\left(B_{n}^{-1} X_{j}-f_{a}\left(B_{n}^{-1} X_{j}\right)\right)=: T_{n, k}^{(a)}+V_{n, k}^{(a)} .
$$

Choose arbitrary $\eta>0$. We have for $a>\eta^{-1 / \alpha}$

$$
\begin{aligned}
\limsup _{n \rightarrow \infty} \mathbb{P}\left(V_{n, k}^{(a)} \neq 0\right) & \leq \limsup _{n \rightarrow \infty} \mathbb{P}\left(\exists 1 \leq j \leq n:\left|X_{j}\right|>a B_{n}\right) \\
& \leq \limsup _{n \rightarrow \infty} n \mathbb{P}\left(\left|X_{1}\right|>a B_{n}\right)=a^{-\alpha}<\eta
\end{aligned}
$$

Consequently

$$
\limsup _{n \rightarrow \infty}\left|E e^{i \lambda S_{n} / B_{n}}-E e^{i \lambda T_{n, n}^{(a)}}\right|<2 \eta .
$$

A similar reasoning also shows that

$$
\limsup _{m \rightarrow \infty}\left|\left(E e^{i \lambda S_{N} / B_{m \cdot N}}\right)^{m}-\left(E e^{i \lambda T_{m \cdot N, N}^{(a)}}\right)^{m}\right|<2 \eta .
$$

It follows that (30) will hold provided for each $a>0$

$$
\lim _{N \rightarrow \infty} \limsup _{m \rightarrow \infty}\left|E e^{i \lambda \sum_{j=1}^{m \cdot N} U_{m \cdot N, j}^{(a)}}-\left(E e^{i \lambda \sum_{j=1}^{N} U_{m \cdot N, j}^{(a)}}\right)^{m}\right|=0,
$$

where

$$
U_{n, j}^{(a)}=f_{a}\left(B_{n}^{-1} X_{j}\right)
$$

Now we are ready to apply Newman's inequality [15] (see also [16, Theorem 1]). Take $\lambda \in \mathbb{R}^{1}$ 
and return for a while to $n=m \cdot N$. Then

$$
\begin{aligned}
& \left|E \exp \left\{i \lambda \sum_{j=1}^{n} U_{n, j}^{(a)}\right\}-\left(E \exp \left\{i \lambda \sum_{j=1}^{N} U_{n, j}^{(a)}\right\}\right)^{m}\right| \\
& \leq \frac{\lambda^{2}}{2} \sum_{1 \leq k \neq l \leq m} \operatorname{Cov}\left(\sum_{i=(k-1) \cdot N+1}^{k \cdot N} U_{n, i}^{(a)}\right)\left(\sum_{j=(l-1) \cdot N+1}^{l \cdot N} U_{n, j}^{(a)}\right) \quad \text { Newman's inequality } \\
& =\frac{\lambda^{2}}{2}\left(\operatorname{Var}\left(\sum_{j=1}^{n} U_{n, j}^{(a)}\right)-m \operatorname{Var}\left(\sum_{j=1}^{N} U_{n, j}^{(a)}\right)\right) \\
& =\frac{\lambda^{2} n}{2}\left(\frac{1}{n} \operatorname{Var}\left(\sum_{j=1}^{n} U_{n, j}^{(a)}\right)-\frac{1}{N} \operatorname{Var}\left(\sum_{j=1}^{N} U_{n, j}^{(a)}\right)\right) \\
& =\lambda^{2}\left\{\sum_{j=2}^{N}\left(\frac{1}{N}-\frac{1}{n}\right)(j-1)\left(n \operatorname{Cov}\left(U_{n, 1}^{(a)}, U_{n, j}^{(a)}\right)\right)\right. \\
& \left.+\sum_{j=N+1}^{n}\left(1-\frac{j-1}{n}\right)\left(n \operatorname{Cov}\left(U_{n, 1}^{(a)}, U_{n, j}^{(a)}\right)\right)\right\} \\
& \leq \lambda^{2}\left\{\frac{1}{N} \sum_{i=1}^{N} \sum_{j=i+1}^{N}\left(n \operatorname{Cov}\left(U_{n, 1}^{(a)}, U_{n, j}^{(a)}\right)\right)+\frac{1}{N} \sum_{i=1}^{N} \sum_{j=N+1}^{n}\left(n \operatorname{Cov}\left(U_{n, 1}^{(a)}, U_{n, j}^{(a)}\right)\right)\right\} \\
& =\lambda^{2} \frac{1}{N} \sum_{i=1}^{N} \sum_{j=i+1}^{n}\left(n \operatorname{Cov}\left(U_{n, 1}^{(a)}, U_{n, j}^{(a)}\right)\right) \\
& \leq \lambda^{2} \frac{1}{N} \sum_{i=1}^{N} n B_{n}^{-2} \sum_{j=i+1}^{\infty} \operatorname{Cov}\left(f_{a \cdot B_{n}}\left(X_{1}\right), f_{a \cdot B_{n}}\left(X_{j}\right)\right) \\
& \underset{m \rightarrow \infty}{\longrightarrow} \lambda^{2} a^{2-\alpha} \frac{1}{N} \sum_{i=1}^{N} \sum_{j=i+1}^{\infty} \int_{\mathbb{R}^{2}} f_{1}\left(x_{1}\right) f_{1}\left(x_{2}\right) \nu_{\{1, j\}}\left(d x_{1}, d x_{2}\right) \underset{N \rightarrow \infty}{\longrightarrow} 0,
\end{aligned}
$$

by (23), (24) and (25).

\subsection{Proof of Theorem 5}

In view of [14, Theorem 1] it is enough to establish the finite dimensional convergence. Since the increments of $\left\{S_{n}(t)\right\}$ are (asymptotically) stationary we need only asymptotic independence of the increments. For the sake of brevity we shall restrict our attention to two adjoining increments $S_{n}\left(t_{1}\right)-S_{n}\left(t_{0}\right)$ and $S_{n}\left(t_{2}\right)-S_{n}\left(t_{1}\right), 0 \leq t_{0}<t_{1}<t_{2} \leq 1$. Let $\lambda, \theta \in \mathbb{R}^{1}$.

In order to prove that

$$
\begin{aligned}
\lim _{n \rightarrow \infty} \mid \mathbb{E} \exp \left(i \lambda \left(S_{n}\left(t_{1}\right)\right.\right. & \left.\left.-S_{n}\left(t_{0}\right)\right)+i \theta\left(S_{n}\left(t_{2}\right)-S_{n}\left(t_{1}\right)\right)\right) \\
& -\mathbb{E} \exp \left(i \lambda\left(S_{n}\left(t_{1}\right)-S_{n}\left(t_{0}\right)\right)\right) \cdot \mathbb{E} \exp \left(i \theta\left(S_{n}\left(t_{2}\right)-S_{n}\left(t_{1}\right)\right)\right) \mid=0
\end{aligned}
$$

we may, as before replace the increments with sums of $U_{n, j}^{(a)}$, for $a>0$ large enough. Then we 
have

$$
\begin{aligned}
\mid \mathbb{E} \exp \{ & \left.i \lambda\left(\sum_{\left\lfloor n t_{0}\right\rfloor<j \leq\left\lfloor n t_{1}\right\rfloor} U_{n, j}^{(a)}\right)+i \theta\left(\sum_{\left\lfloor n t_{1}\right\rfloor<j \leq\left\lfloor n t_{2}\right\rfloor} U_{n, j}^{(a)}\right)\right\} \\
& -\mathbb{E} \exp \left\{i \lambda\left(\sum_{\left\lfloor n t_{0}\right\rfloor<j \leq\left\lfloor n t_{1}\right\rfloor} U_{n, j}^{(a)}\right)\right\} \cdot \mathbb{E} \exp \left\{i \theta\left(\sum_{\left\lfloor n t_{1}\right\rfloor<j \leq\left\lfloor n t_{2}\right\rfloor} U_{n, j}^{(a)}\right)\right\} \mid \\
& \leq|\lambda||\theta| \operatorname{Cov}\left(\sum_{\left\lfloor n t_{0}\right\rfloor<j \leq\left\lfloor n t_{1}\right\rfloor} U_{n, j}^{(a)}, \sum_{\left\lfloor n t_{1}\right\rfloor<k \leq\left\lfloor n t_{2}\right\rfloor} U_{n, j}^{(a)}\right) \quad \text { Newman's inequality } \\
& \leq|\lambda||\theta| \sum_{r=1}^{n-1} r \cdot \operatorname{Cov}\left(U_{n, 1}^{(a)}, U_{n, 1+r}^{(a)}\right) \\
& \leq|\lambda||\theta|\left\{\frac{M}{n} \sum_{r=1}^{M} n B_{n}^{-2} \operatorname{Cov}\left(f_{a \cdot B_{n}}\left(X_{1}\right), f_{a \cdot B_{n}}\left(X_{1+r}\right)\right)\right. \\
& \left.+\sum_{r=M+1}^{\infty} n B_{n}^{-2} \operatorname{Cov}\left(f_{a \cdot B_{n}}\left(X_{1}\right), f_{a \cdot B_{n}}\left(X_{1+r}\right)\right)\right\} \\
& \underset{n \rightarrow \infty}{\longrightarrow}|\lambda||\theta| a^{2-\alpha} \sum_{r=M+1}^{\infty} \int_{\mathbb{R}^{2}} f_{1}\left(x_{1}\right) f_{1}\left(x_{2}\right) \nu_{\{1,1+r\}}\left(d x_{1}, d x_{2}\right) \underset{M \rightarrow \infty}{\longrightarrow} 0,
\end{aligned}
$$

again by (23), (24) and (25).

\section{References}

[1] F. Avram and M.S. Taqqu, Weak convergence of sums of moving averages in the $\alpha$-stable domain of attraction, Ann. Probab., 20:483-503, 1992.

[2] B. Basrak, R.A. Davis, and T. Mikosch, A characterization of multivariate regular variation, Ann. Appl. Probab., 12:908-920, 2002.

[3] B. Basrak and J. Segers, Regularly varying multivariate time series, Stochastic Process. Appl., 119:1055-1080, 2009.

[4] A. Bulinski and A. Shashkin, Limit theorems for associated random fields and related systems, World Scientific Publishing, Singapore, 2007.

[5] R.M. Burton, A.R. Dabrowski, and H. Dehling, An invariance principle for weakly associated random vectors, Stochastic Process. Appl., 23:301-306, 1986.

[6] A.R. Dabrowski and A. Jakubowski, Stable limits for associated random variables, Ann. Probab., 22(1):1-16, 1994.

[7] J. Damarackas and V. Paulauskas, Properties of spectral covariance for linear processes with infinite variance, Lith. Math. J., 54(3):252-276, 2014.

[8] J. Damarackas and V. Paulauskas, Spectral covariance and limit theorems for random fields with infinite variance, J. Multivariate Anal., 153:156-175, 2017. 
[9] J. Esary, F. Proschan, and D.J. Walkup, Association of random variables with applications, Ann. Math. Statist., 38:1466-1474, 1967.

[10] W. Feller, An Introduction to Probability Theory and Its Applications, Vol. II, 2nd Ed., Wiley, New York, 1971.

[11] Ch. Houdré, V. Pérez-Abreu, and D. Surgailis, Interpolation, correlation identities, and inequalities for infinitely divisible variables, J. Fourier Anal. Appl., 4:651-668, 1998.

[12] J. Jacod and A.N. Shiryayev, Limit Theorems for Stochastic Processes, Springer, Berlin, 1987.

[13] M-L.T. Lee, S.T. Rachev, and G. Samorodnitsky, Association of stable random variables, Ann. Probab., 18:1759-1764, 1990.

[14] S. Louhichi and E. Rio, Functional convergence to stable Lévy motions for iterated random Lipschitz mappings, Electron. J. Probab., 16:2452-2480, 2011.

[15] C.M. Newman, Normal fluctuations and the FKG inequalities, Comm. Math. Phys., 74:119-128, 1980.

[16] CM. Newman and A.L.J. Wright, An invariance principle for certain dependent sequences, Ann. Probab., 9:671-675, 1981.

[17] V. Paulauskas, Some remarks on multivariate stable distributions, J. Multivariate Anal., 6:356-368, 1976.

[18] V. Paulauskas, On $\alpha$-covariance, long, short, and negative memories for sequences of random variables with infinite variance, ArXiv, 2013, available from: https://arxiv.org/abs/1311.0606v1

[19] V. Paulauskas, A note on linear processes with tapered innovations, Lith. Math. J., 2019, available from: https://doi.org/10.1007/s10986-019-09445-w.

[20] S.I. Resnick, Point processes, regular variation and weak convergence, Adv. in Appl. Probab., 18:66-138, 1986.

[21] S.I. Resnick, Association and multivariate extreme value distributions, in C.C. Heyde (Ed.), Gani Festschrift: Studies in Statistical Modeling and Statistical Science. year, Statist. Soc. of Australia, 1988.

[22] G. Samorodnitsky, Association of infintely divisible random vectors, Stochastic Process. Appl., 55:45-55, 1995.

[23] G. Samorodnitsky and M.S. Taqqu, Stable Non-Gaussian Random Processes, Chapman \& Hall, New York, 1994.

[24] A. V. Skorohod, Limit theorems for stochastic processes, Theory Probab. Appl., 1:261290, 1956.

[25] W. Whitt, Stochastic-Process Limits. An introduction to Stochastic-Process Limits and Their Application to Queues, Springer, New York, 2002. 
[26] H. Yu, A Glivenko-Cantelli lemma and an invariance principle for empirical processes of associated sequences, Probab. Theory Related Fields, 95:357-370, 1993. 